\title{
A Durable 4-1BB-based CD19 CAR-T Cell for Treatment of Relapsed or Refractory Non-Hodgkin Lymphoma
}

\section{Zhitao Ying}

Peking University Cancer Hospital \& Institute

Ting He

Immunochina Pharmaceuticals Co., Ltd

Shanzhao Jin

Immunochina Pharmaceuticals Co., Ltd

\section{Xiaopei Wang}

Peking University Cancer Hospital \& Institute

Wen Zheng

Peking University Cancer Hospital \& Institute

Ningjing Lin

Peking University Cancer Hospital \& Institute

Meifeng Tu

Peking University Cancer Hospital \& Institute

Yan Xie

Peking University Cancer Hospital \& Institute

\section{Lingyan Ping}

Peking University Cancer Hospital \& Institute

Weiping Liu

Peking University Cancer Hospital \& Institute

\section{Lijuan Deng}

Peking University Cancer Hospital \& Institute

\section{Yanping Ding}

Immunochina Pharmaceuticals Co., Ltd

\section{Xuelian Hu}

Immunochina Pharmaceuticals Co., Ltd

\section{Bing Bu}

Shandong Academy of Medical Sciences

Xin-an Lu ( $\sim$ LXA2020@aliyun.com ) Immunochina Pharmaceuticals Co., Ltd

\section{Yuqin Song}


Peking University Cancer Hospital \& Institute

Jun Zhu

Peking University Cancer Hospital \& Institute

\section{Research Article}

Keywords: CD19 CAR-T, 4-1BB, safety, durable efficacy

Posted Date: October 8th, 2021

DOl: https://doi.org/10.21203/rs.3.rs-846294/v1

License: (c) (1) This work is licensed under a Creative Commons Attribution 4.0 International License. Read Full License

Version of Record: A version of this preprint was published at Chinese Journal of Cancer Research on January 1st, 2022. See the published version at https://doi.org/10.21147/j.issn.1000-9604.2022.01.05. 


\section{Abstract \\ Backgroud}

: CD19-directed chimeric antigen receptor $T$ (CAR-T) cell therapy has shown great promise for cure of relapsed or refractory B cell non-Hodgkin lymphoma ( $r / r$ B-NHL). Previous studies reported that 4-1BBbased CD19 CAR-T was more beneficial for the clinical outcomes than CD28-based CAR-T, especially lower rates of severe adverse events. However, the median progression-free survival (mPFS) of 4-1BBbased product Kymriah was shorter than that of the CD28-based Yescarta (2.9 v.s. 5.9 months), suggesting that the long-term effectiveness of Kymriah was limited. Thus, a safe and durable 4-1BBbased CD19 CAR-T needs to be developed.

\section{Methods}

Here we presented a CD19 CAR-T (named IM19) with FMC63 SCFv, 4-1BB and CD3 3 intracellular domain, which was manufactured into a memory T-enriched formulation and launched a clinical trial for treatemt of r/r B-NHL. The clinical outcomes of IM19 were evaluated in $22 \mathrm{r} / \mathrm{r} \mathrm{B}-\mathrm{NHL}$ patients in a Phase I/II trial with dose-escalation investigation $\left(5 \times 10^{5}, 1 \times 10^{6}\right.$ and $\left.3 \times 10^{6} / \mathrm{kg}\right)$.

\section{Results}

All patients received a single infusion of IM19 after 3-day conditional regimen. Cytokine release syndrome (CRS) occurred in 13 patients (59\%), with $54.5 \%$ of grade $1-2$ and $4.5 \%$ of grade 3 , whereas Kymriah led to $22 \%$ of $>$ grade 3 CRS. Only one patient showed > grade 3 immune effector cell-associated neurotoxicity syndrome (ICANS). At 6 months, the overall response rate was $47.62 \%$, and the complete response rate was $42.86 \%$. The mPFS of IM19 achieved 9 months and the 1 -year overall survival rate was $78 \%$.

\section{Conclusions}

These results demonstrated the safety and durable efficacy of IM19 with FMC63 scFv, 4-1BB and CD3Z intracellular domain, that is promising for further development and clinical investigation.

\section{Trial registration}

: The registration date of the clinical trial is May $17,2018(17 / 05 / 2018)$ and the trial registration numbers is NCT03528421.

\section{Introduction}


Non-Hodgkin lymphoma $(\mathrm{NHL})$ is one of the most common cancer types around the world, accounting for about $2.8 \%$ of new cancer cases and $2.6 \%$ of the cancer deaths in 2018 [1]. There were 68,500 new cases and 37,600 deaths of NHL in China from 2006 to 2016 [2], and both the incidence and mortality of NHL in male had ranked top 10th since 2018 [3]. As one of the most common hematologic malignancies, approximately two thirds of patients could be cured with standard frontline therapy. However, it is difficult to manage the disease when it progressed to the relapsed or refractory $(r / r)$ stage [4]. The standard of care therapy for $r / r$ NHL patients was salvage chemotherapy followed by an autologous stem cell transplant (ASCT) [5]. Although $50 \%-60 \%$ of $\mathrm{NHL}$ patients could be eligible for an ASCT, about $40 \%-50 \%$ tend to relapse [6]. Thus, more effective new therapies are needed for patients with $r / r$ NHL.

CD19-directed chimeric antigen receptor-T (CAR-T) cell therapy has shown remarkable antitumor activity in patients with $r / r$ hematologic malignancies in several multicenter clinical studies [7]. Currently, three types of CD19 CAR-T cell therapies for B cell lymphoma have been approved all over the world, including Yescarta (axicabtagene-ciloleucel, axi-cel), Kymriah (tisagenlecleucel, tisa-cel) and Breyanzi (lisocabtagene-maraleucel, liso-cel) [8-11]. Although these three products used the same single chain fragment variable (scFv) that targeted the extracellular domain of CD19 antigen, their safety and efficacy profiles were different, partially owing to the variations in co-stimulatory domain and manufacturing technologies. The pivotal ZUMA-1 trial for axi-cel showed that the overall response rate (ORR) was $83 \%$ with $58 \%$ of complete remission (CR) at one month, and the median progression free survival (mPFS) time was 5.9 months [12]. In the JULIET study for tisa-cel, the ORR and CR were $52 \%$ and $40 \%$ at one month, respectively, and the mPFS was 2.9 months [13]. These data suggested that both the initial response and long-term efficacy of the 4-1BB-based product tisa-cel were limited and needed to be improved.

Besides therapeutic efficacy, the safety profiles of three products especially severe adverse event rates were distinct. The most common CAR-T cell toxicities included cytokine release syndrome (CRS) and immune effector cell-associated neurotoxicity syndrome (ICANS). The axi-cel with the CD28 costimulatory molecule showed $93 \%$ incidence of CRS with $13 \%$ of > grade 3 [14], while the $4-1 \mathrm{BB}$ costimulated liso-cel induced $37 \%$ of CRS with $1 \%$ of > grade 3 [11], and tisa-cel resulted in $58 \%$ of CRS with $22 \%$ of $>$ grade 3 [15]. Regarding of the $>$ grade 3 ICANS, the incidence rate was $32 \%$ for axi-cel, whereas $12 \%$ for tisa-cel and $10 \%$ for liso-cel $[11,14,15]$. Overall, the probabilities of severe CRS and ICANS were higher from axi-cel compared to those from tisa-cel and liso-cel [16]. These clinical investigations indicate that 4-1BB domain may endow CD19 CAR-T with a better safety profile than CD28 domain. Recently, we have also compared the efficacy and adverse events of CD19 CAR-T cells with CD28 or 4-1BB costimulatory domain in a small cohort of $r / r$ B-NHL patients, and confirmed that 4-1BB signaling was superior to CD19 CAR-T therapy in safety [17]. Similar clinical outcomes were observed in B-cell acute lymphoblastic leukemia patients [18]. Thus, compared with CD28-based CAR-T, 4-1BB-based CD19 CAR-T exhibited better safety for B-NHL therapy, however, the durable efficacy needs to be warranted.

Based on previous reports, we designed a 4-1BB-based CD19 CAR-T (named IM19) that was composed of the FMC63 scFv, the hinge and transmembrane domain of CD8a, the 4-1BB co-stimulatory domain, and 
the $\mathrm{CD} 3 \zeta$ intracellular domain, and manufactured the CAR-T cells into a memory cell-enriched formulation. We systemically investigated the safety and efficacy of IM19 in $22 \mathrm{r} / \mathrm{r}$ B-NHL patients of a Phase I/II trial. Our clinical data demonstrated that IM19 showed great potential in safety and long-term efficacy for $\mathrm{r} / \mathrm{r}$ B-NHL therapy.

\section{Material And Methods}

\subsection{Patient eligibility criteria}

Patients with CD19-positive r/r B-NHL who previously received at least one line of therapy were recruited (Supplementary Table S1). Subject inclusion criteria include: 1 ) age $\geq 18$ years; 2 ) patients with evaluable disease lesions; 3 ) expected life span of over 90 days; 4) an Eastern Cooperative Oncology Group (ECOG) score of 0-2 points; and 5) women of childbearing age with a negative blood pregnancy test. Exclusion criteria are as follows: 1 ) patients with a history of central nervous system disease, or graft-versus-host disease; 2) patients who received any chemotherapy or radiotherapy within 3 days, or systemic steroids within 5 days before apheresis; 3 ) patients who received any drug of stimulating bone marrow growing within 5 days before apheresis; or 4) patients who received any gene cell therapy.

\subsection{Clinical study design}

The clinical trial (ClinicalTrials.gov identifier: NCT03528421) was a single center phase I/II study to evaluate the safety and efficacy of IM19 in patients with r/r B-NHL [17]. This study recruited 26 patients in total. Four cases were excluded according to the patient eligibility criteria (Fig. $1 \mathrm{~A}$ ). To assess the doselimiting toxicity (DLT) of IM19, three dose levels of $5 \times 10^{5} / \mathrm{kg}, 1 \times 10^{6} / \mathrm{kg}$, and $3 \times 10^{6} / \mathrm{kg}$ were assessed with 3, 6 and 6 patients in each cohort (Table 1). Another 7 patients were enrolled for extensive investigation at the dose level $5 \times 10^{5} / \mathrm{kg}$ (Table 1 and Fig. $1 \mathrm{~A}$ ). Once DLT occurred in more than 2 cases, the recruitment should be terminated at this dose. This study was conducted in accordance with the Declaration of Helsinki and was approved by the Institutional Review Board of Peking University Cancer Hospital (IRBPUCH). All participants were informed of the possible risks and side effects of the therapy and provided signed informed consent. 
Table 1

Patients and treatment characteristics

\begin{tabular}{|c|c|c|c|c|c|c|c|c|}
\hline ID & Sex & $\begin{array}{l}\text { Age } \\
\text { (year) }\end{array}$ & $\begin{array}{l}\text { Weight } \\
\text { (kg) }\end{array}$ & Diagnosis & $\begin{array}{l}\text { ECOG } \\
\text { PS }\end{array}$ & $\begin{array}{l}\text { Prior lines of } \\
\text { therapy }\end{array}$ & $\begin{array}{l}\text { Dose } \\
\text { level }\end{array}$ & $\begin{array}{l}\text { BTD } \\
(S P D, \\
\left.\mathrm{mm}^{2}\right)\end{array}$ \\
\hline F0101 & Male & 49 & 70 & MZL & 0 & 2 & $5 \times 10^{5} / \mathrm{kg}$ & 814 \\
\hline F0102 & Male & 34 & 78 & DLBCL & 0 & 1 & $5 \times 10^{5} / \mathrm{kg}$ & 1044 \\
\hline F0103 & Male & 59 & 92 & $\begin{array}{l}\text { FL (Grade } \\
\text { 2) }\end{array}$ & 0 & $>2$ & $5 \times 10^{5} / \mathrm{kg}$ & 7472 \\
\hline F0104 & Male & 65 & 73 & $\begin{array}{l}\text { FL (Grade } \\
\text { 2) }\end{array}$ & 0 & $>2$ & $1 \times 10^{6} / \mathrm{kg}$ & 3679 \\
\hline F0106 & Female & 50 & 57 & DLBCL & 0 & $>2$ & $1 \times 10^{6} / \mathrm{kg}$ & 1829 \\
\hline F0107 & Female & 36 & 48 & $\begin{array}{l}\text { FL (Grade } \\
\text { 2) }\end{array}$ & 0 & 2 & $1 \times 10^{6} / \mathrm{kg}$ & 1910 \\
\hline F0109 & Male & 65 & 88 & DLBCL & 0 & $>2$ & $1 \times 10^{6} / \mathrm{kg}$ & 11246 \\
\hline F0110 & Male & 61 & 67 & MZL & 0 & $>2$ & $1 \times 10^{6} / \mathrm{kg}$ & 1534 \\
\hline F0111 & Female & 49 & 76 & DLBCL & 0 & $>2$ & $1 \times 10^{6} / \mathrm{kg}$ & 1692 \\
\hline F0112 & Male & 59 & 76 & $\begin{array}{l}\text { FL (Grade } \\
\text { 3a) }\end{array}$ & 0 & 1 & $3 \times 10^{6} / \mathrm{kg}$ & 7108 \\
\hline F0113 & Female & 47 & 54 & DLBCL & 0 & 2 & $3 \times 10^{6} / \mathrm{kg}$ & 628 \\
\hline F0114 & Male & 29 & 78 & $\begin{array}{l}\text { FL (Grade } \\
\text { 2) }\end{array}$ & 0 & 2 & $3 \times 10^{6} / \mathrm{kg}$ & 2068 \\
\hline F0115 & Male & 54 & 82 & DLBCL & 0 & 2 & $3 \times 10^{6} / \mathrm{kg}$ & 2776 \\
\hline F0116 & Male & 62 & 72 & TFL & 0 & 2 & $3 \times 10^{6} / \mathrm{kg}$ & - \\
\hline F0117 & Male & 28 & 97 & DLBCL & 0 & $>2$ & $3 \times 10^{6} / \mathrm{kg}$ & 1901 \\
\hline F0118 & Male & 48 & 80 & DLBCL & 0 & $>2$ & $1 \times 10^{6} / \mathrm{kg}$ & 1492 \\
\hline F0119 & Female & 61 & 62 & $\begin{array}{l}\text { FL (Grade } \\
3 b)\end{array}$ & 0 & $>2$ & $1 \times 10^{6} / \mathrm{kg}$ & 2109 \\
\hline F0121 & Female & 69 & 62 & DLBCL & 1 & 2 & $1 \times 10^{6} / \mathrm{kg}$ & 2002 \\
\hline F0122 & Female & 65 & 62 & DLBCL & 0 & 2 & $1 \times 10^{6} / \mathrm{kg}$ & 9208 \\
\hline \multicolumn{9}{|c|}{$\begin{array}{l}\text { Abbreviations: MZL, marginal zone B-cell lymphoma; DLBCL, diffuse large B cell lymphoma; FL, } \\
\text { follicular lymphoma; ECOG PS: Eastern Cooperative Oncology Group performance status; BTD: } \\
\text { Baseline tumor burden; SPD: sum of the products of diameters. }\end{array}$} \\
\hline
\end{tabular}




\begin{tabular}{|c|c|c|c|c|c|c|c|c|}
\hline ID & Sex & $\begin{array}{l}\text { Age } \\
\text { (year) }\end{array}$ & $\begin{array}{l}\text { Weight } \\
\text { (kg) }\end{array}$ & Diagnosis & $\begin{array}{l}\text { ECOG } \\
\text { PS }\end{array}$ & $\begin{array}{l}\text { Prior lines of } \\
\text { therapy }\end{array}$ & $\begin{array}{l}\text { Dose } \\
\text { level }\end{array}$ & $\begin{array}{l}\text { BTD } \\
(\mathrm{SPD}, \\
\left.\mathrm{mm}^{2}\right)\end{array}$ \\
\hline F0123 & Female & 55 & 63 & DLBCL & 1 & 2 & $1 \times 10^{6} / \mathrm{kg}$ & 8508 \\
\hline F0125 & Male & 62 & 71 & DLBCL & 0 & 2 & $1 \times 10^{6} / \mathrm{kg}$ & 5490 \\
\hline F0126 & Male & 45 & 68 & DLBCL & 0 & 2 & $1 \times 10^{6} / \mathrm{kg}$ & 3717 \\
\hline
\end{tabular}

A schematic design of the clinical trial is shown in Fig. 1B. Apheresis was performed in all eligible patients for production of CAR-T cells. After pre-treated with fludarabine $\left(25 \mathrm{mg} / \mathrm{m}^{2} /\right.$ day $)$ and cyclophosphamide ( $250 \mathrm{mg} / \mathrm{m}^{2} /$ day) for 3 consecutive days, patients were infused with IM19 on day 0 . The primary endpoints were 3-month ORR, and the incidence of DLT. CR, partial remission and ORR at day 28 and month 6 were secondary objectives. The adverse events including routine blood analysis, blood biochemistry, $C$ reactive protein (CRP), CRS, and ICANS were closely monitored especially within one months. On day $-1,4,7,10,14,21,28,90$, and 180 , the peripheral blood samples were collected, and the ratio of IM19 and level of cytokines were detected using flow cytometry.

\subsection{Vector construction and lentivirus production}

The molecular structure of IM19 was comprised of the FMC63-derived ScFv, the hinge and transmembrane domains of CD8a, and the intracellular domains of 4-1BB and CD3ろ. The IM19 gene was ligated to an EF1 a promoter-based lentiviral transfer plasmid pLenti6.3/V5 (ThermoFisher, Waltham, MA, USA). The transfer plasmid, packaging plasmids (pLP1 and pLP2, ThermoFisher, Waltham, MA, USA) and envelope plasmid ( $\mathrm{pLP} / \mathrm{VSVG}$, ThermoFisher, Waltham, MA, USA) were co-transfected into suspension 293 cells using polyethyleneimine (Polysciences, Warrington, PA, USA). After purified from the culture medium using Core 700 chromatography (GE Healthcare, Chicago, IL, USA), the IM19 lentiviral vectors were formulated, cryopreserved, and sent for quality control in Immunochina Pharmaceuticals Co., Ltd. (Beijing, China).

\subsection{Manufacture of IM19 CAR-T cells}

IM19 CAR-T cells were manufactured by Immunochina Pharmaceuticals Co., Ltd. (Beijing, China). Briefly, peripheral blood mononuclear cells (PBMCs) were isolated from the apheresis products using a density gradient medium Ficoll (GE Healthcare, Chicago, IL, USA). T cells were purified and activated by immunomagnetic CD3/CD28 Dynabeads (ThermoFisher, Waltham, MA, USA) at a T cell/bead ratio of 1:1. One day later, T cells were transduced with the IM19 lentiviral vector and cultured in X-VIVO 15 medium (Lonza Group, Basel, Switzerland) with $100 \mathrm{U} / \mathrm{mL}$ of IL-2 at a density of $1.5 \times 10^{6} / \mathrm{mL}$ for $7-11$ days. Then CAR-T cells were formulated, cryopreserved, and sent for quality control in Immunochina Pharmaceuticals Co. Ltd. 


\subsection{Flow cytometry analysis of CAR-T cells}

Flow cytometry analysis was performed using NovoCyte 2060R (ACEA Biosciences, San Diego, CA, USA) according to previous report [17]. The transduction efficiency of IM19 gene into T cells was detected using a phycoerythrin (PE)-labeled anti-CAR antibody that recognized the scFv of IM19 and was developed by Immunochina Pharmaceuticals. PE-anti-CD 4 and FITC-anti-CD8 antibodies (BD Biosciences, San Jose, CA, USA) were used to detect the CD4/CD8 ratio. The APC-anti-CD45RA and FITC-anti-CD62L antibodies (Biolegend, San Diego, CA, USA) were used to evaluate the ratio of memory $T$ cells.

\subsection{Cytometric bead array assay of cytokines}

The blood samples were harvested at the follow-up time points. The protein level of interleukins (IL-2, IL-4, IL-6, IL-10, and IL-17A), interferon (IFN)- $y$ and tumor necrosis factor (TNF) in each sample were evaluated using the Cytometric Bead Array (CBA) Human Th1/Th2/Th17 Cytokine Kit (BD Biosciences, San Jose, CA, USA) and flow cytometry analysis. All assays were carried out according to the manufacturer's protocol. Flow cytometry was used to detect the cytokine levels in serum samples. The data were expressed as picograms per milliliter of serum.

\subsection{Evaluation of clinical outcomes}

The patients were followed up and adverse events were evaluated according to the clinical symptoms, routine blood analysis, blood biochemistry, and immune indexes. Neurotoxicity was graded according to National Cancer Institute ( $\mathrm{NCl}$ ) Common Terminology Criteria for Adverse Events (CTCAE). CRS was assessed in line with the American Society for Blood and Marrow Transplantation (ASBMT) Consensus Criteria. The tumor burden was assessed using PET-CT for response evaluation based on the revised criteria for response assessment [19].

\subsection{Statistical analysis}

Statistical analyses were performed with GraphPad Prism (version 8.0, GraphPad Software, Inc., La Jolla, CA, USA) and SPSS (version 22.0, IBM Corp., New York, USA) software. To compare two groups, we used the two-sided Wilcoxon-Mann-Whitney test. For three or more groups, statistical analysis was based on one-way ANOVA and Tukey's multiple comparison test. A threshold of $P<0.05$ was considered statistically significant for all analyses.

\section{Results}

\subsection{Study design and characteristics of patients}

A single center Phase I/II study was initiated to assess the safety and efficacy of IM19. From June 2018 to October 2019, a total of 26 patients with r/r B-NHL were recruited. CD19 antigen expression was identified by immunohistochemistry. Of these, 22 patients ranging from 27 to 69 years old, were eligible to be enrolled (Table 1). A dose-escalation study was first performed to evaluate the DLT and best dose in 
15 patients using the dose level of $5 \times 10^{5} / \mathrm{kg}$ (3 patients), $1 \times 10^{6} / \mathrm{kg}$ (6 patients), and $3 \times 10^{6} / \mathrm{kg}(6$ patients). Then an extensive investigation of the best dose was carried out in 7 patients (Table 1). The basic characteristics of patients were summarized in Table 1, and the features of patients in each dose cohort were analyzed in Supplementary Table S1. Three lymphoma types including MZL (2/22), DLBCL $(13 / 22)$ and FL (7/22) were present in each dose cohort. About $90.9 \%(20 / 22)$ of patients received at least 2 lines of prior therapies before enrollment.

Apheresis of the enrolled patients was performed and transported to the GMP facility of Immunochina Pharmaceuticals Co. Ltd. After PBMCs were isolated from the leukapheresis, T cells were purified, activated, and transduced with the IM19 lentiviral vectors (Fig. 1B). The IM19 CAR-T cells were cultured in serum-free medium. When expanding to the dose level, cells were evaluated for the CAR expression ratio, CD4/CD8 ratio, and the percentage of memory T cells (Supplementary Table S2). Then IM19 CAR-T cells were formulated and underwent quality control. Under our manufacturing process, the percentage of naïve, stem cell memory, central memory, and effector memory T cells in almost all final IM19 products remained at high levels (Supplementary Table S2). On day 0, patients were infused with IM19 CAR-T cells intravenously, and were closely followed up from day 4 to day 28 for safety evaluation (Fig. 1B). The response was assessed at day 28,90 , and 180 (Fig. 1B).

\subsection{Safety of IM19 CAR-T cells}

As of March 1st, 2020, data cut-off, 22 patients were infused with IM19 CAR-T cells. The adverse events associated with CAR-T cells were monitored from day 0 to day 28 . CRS was observed in 59\% (13/22) patients among whom one case (4.5\%) showed both grade 3 CRS and grade 3 ICANS at the dose of $3 \times 10^{6} / \mathrm{kg}$ (Fig. 2A, Supplementary Table S3). According to the guidelines, $400 \mathrm{mg}$ of tocilizumab and 10 $\mathrm{mg}$ of dexamethasone were used for the toxicity management. All the adverse events were controlled within 2 months. All patients showed tolerability to IM19 at three dose levels, and DLT was not reached.

To further evaluate the adverse events, we assessed the levels of blood routine, blood chemistry, and blood inflammatory cytokines at the follow-up time point. Almost all patients experienced cytopenia and 8 patients showed decrease in platelet after IM19 treatment. Multiple cytokines especially IL-6, IL-10 and IFN- $y$ increased in 12 patients from day 4 to 28 (Fig. 2B-D). The patient who displayed severe CRS and ICANS also showed much higher levels of IL-6, IL-10 and IFN-y (Fig. 2A-D, Supplementary Table S3).

\subsection{Clinical responses of IM19 CAR-T cells}

One month and three months after IM19 treatment, patients received PET-CT scanning for analysis of tumor burden. Decrease in sum of the products of diameters (SPD) was observed in 16 patients, and the ORR was $72.7 \%$ and CR was $40.9 \%$ at day 28 (Fig. 3A-B, Table 2). The 3-month CR was $50.0 \%$, and the 6month CR was $40.9 \%$ (Table 2). Seven patients (31.8\%) showed ongoing response even after 1 year (Fig. $3 A$ ). Evident eradication of tumors and durable effectiveness was observed in the CR patients, and representative PET-CT images were shown in Fig. 3C. Six patients were not responsive to IM19 CAR-T cells and were evaluated as progressive disease at day 28 (Fig. 3A and B). For patients with aggressive 
$\mathrm{NHL}$, the ORR was $62.5 \%(10 / 16)$ and CR was $31.2 \%(5 / 16)$ at day 28 (Table 2). The median PFS was 9 months after IM19 infusion (Fig. 3D), and 1-year overall survival (OS) was 78\% (Fig. 3E).

Table 2

The response rates of 22 patients after IM19 CAR-T cell infusion

\begin{tabular}{|llllllll|}
\hline Groups & & \multicolumn{2}{l}{ 28 days } & \multicolumn{2}{l}{3 months } & \multicolumn{2}{l|}{6 months } \\
\cline { 2 - 8 } & & CR & ORR & CR & ORR & CR & ORR \\
\hline Aggressive NHL & $5 \times 10^{5} / \mathrm{kg}$ & $0 / 1$ & $0 / 1$ & $0 / 1$ & $0 / 1$ & $0 / 1$ & $0 / 1$ \\
\cline { 2 - 8 } & $1 \times 10^{6} / \mathrm{kg}$ & $4 / 10$ & $7 / 10$ & $4 / 10$ & $4 / 10$ & $4 / 10$ & $4 / 10$ \\
\cline { 2 - 8 } & $3 \times 10^{6} / \mathrm{kg}$ & $1 / 5$ & $3 / 5$ & $2 / 5$ & $3 / 5$ & $2 / 5$ & $2 / 5$ \\
\hline All patients & Total & $5 / 16$ & $10 / 16$ & $6 / 16$ & $7 / 16$ & $6 / 16$ & $6 / 16$ \\
\hline
\end{tabular}

\subsection{Correlation of IM19 CAR-T characteristics with clinical outcomes}

We further evaluated the potential factors which were associated with the safety and clinical responses. The levels of IM19 CAR-T cells in peripheral blood were analyzed at the follow-up time point. All patients showed in vivo expansion and high levels of IM19 from day 4 to 14, and then the level decreased, however, persisted at a low expression. The IM19 CAR-T could be detected in some of the subjects even over a period of 8 months (Fig. 4A). Both the ratio of CD4/CD8 in final IM19 products and the level of CAR-T cells in peripheral blood were not significantly correlated with the CRS grades (Fig. 4B).

Importantly, the clinical responses (CR vs PR; PR vs PD) at 3 months were apparently associated with the peak level of CAR-T cells in peripheral blood, although there were only 2 patients in the PR group (Fig. 4C). Particularly, the median AUC-28 (area under the curve of IM19 levels within 28 days) in the group of CR and PR patients was more than 10-fold higher than that in PD group (Fig. 4D). In addition, the transduction efficiency of CAR and the CD4/CD8 ratio in final IM19 products showed no distinct correlation with clinical responses at 1 month or 3 months (Fig. 4C), and they were consistently not correlated with the CAR-T cell peak level in peripheral blood (Fig. 4E).

\section{Discussion}

Anti-CD19 CAR-T cells have demonstrated impressive clinical outcomes in treating B cell malignancies, but the functions of CAR-T cells incorporating with different co-stimulatory domains vary in toxicity, safety and persistence. It has been reported that 4-1BB-based CAR-T cells tended to induce a central memory phenotype, possibly due to the enhanced fatty acid metabolism $[20,21]$. Although previous 
studies showed that 4-1BB signaling was beneficial for the safety and in vivo persistence of CD19 CAR-T cells, the durable effectiveness of 4-1BB-based CD19 CAR-T cells should be paid attention based on the reported clinical data $[13,22]$. In the study, we developed a CD19 CAR-T with 4-1BB domain, and systemically investigated the response and long-term outcomes in $\mathrm{r} / \mathrm{r} \mathrm{B}$-cell $\mathrm{NHL}$ patients. Compared to the CD19 CAR-T products approved by US FDA $[8,9,11]$, our CD19 CAR-T cells showed much longer mPFS and lower rates of ICANS in r/r B-NHL patients. Kymriah showed $58 \%$ of best $C R, 22 \%$ of $\geq$ grade 3 CRS, and $11 \%$ of $\geq$ grade 3 ICANS in NHL patients [22]. In Phase II trial of ZUMA-1, Yescarta-related severe CRS and ICANS were observed in $11 \%$ and $32 \%$ of patients, respectively, and the ORR of Yescarta was $83 \%$ and CR was $58 \%$ [12]. KTE-19 showed ORR was $71 \%$ (5/7) and CR was 57\% (4/7) in Phase I trial of ZUMA-1. Recently approved Breyanzi achieved an ORR of 73\% (186/256) and CR of 53\% (136/256) [11]. Severe CRS and ICANS occurred in 2\% (6/256) and 10\% (27/256) of patients who received Breyanzi [11]. Moreover, the median PFS was 2.9 months for Kymriah, 5.9 months for Yescarta, and 6.8 months for Breyanzi.

In comparison, our IM19 CAR-T cells showed only $4.5 \%$ of severe CRS and ICANS, indicating the good safety profile of this 4-1BB-based CD19 CAR-T. Importantly, the mPFS of IM19 achieved 9 months, which was extremely longer than all three approved products. Our investigation demonstrated that IM19 was a safe and long-term effective CD19 CAR-T. The striking clinical outcomes of IM19 was probably attributed to our knowhow manufacturing technology that generated a $\mathrm{T}$ cell formulation enriched in naïve and memory T cell phenotype (Supplementary Table S2). Gargett et al., have reported that the manufacturing conditions for CAR-T cells could affect the phenotype and functions of CAR-T cells [23], supporting the importance of manufacturing technology and our findings. Moreover, our data showed that the clinical responses were correlated with the in vivo expansion kinetics and peak level of IM19 cells. The majority of T cells in IM19 products skewed toward to high frequency of central memory, effector memory, naïve and stem-like memory T cells (Supplementary Table S2). These superior phenotypes of CAR-T cell products have been reported to be correlated with sustained remissions in patients [24]. Recent studies have revealed that a long-lived CAR-T cells possessing a more naïve/stem/central memory-like T cells (Tscm) / Tcm phenotype achieved optimate control in tumors [21, 25, 26]. Thus, the enriched proportion of naïve and memory T cell phenotypes in IM19 formulation was probably attributed to the in vivo persistence and long-term efficacy.

In addition to the memory phenotype, the proportion of CD4/CD8 in CAR-T cells was also paid attention in previous studies. It was demonstrated that a defined ratio of $\mathrm{CD} 4^{+}$and $\mathrm{CD} 8^{+}$subset of CAR-T cells conferred superior antitumor activity in patients with $\operatorname{ALL}$ and B-NHL $[25,27,28]$. However, our study did not find significant correlation between CD4/CD8 ratio and clinical outcomes including CRS severity, clinical response, and in vivo CAR-T kinetics (Fig. 4B-C, E).

In conclusion, our results demonstrated that IM19 with 4-1BB-based costimulatory domain showed effective and durable antitumor activity, however, very low toxicities for B cell lymphoma. The clinical responses were associated with the in vivo expansion profile of CAR-T cells. Taken together, our work suggested that IM19 was valuable for further development and clinical application. 


\section{Conclusions}

These results demonstrated the safety and durable efficacy of IM19 with FMC63 scFv, 4-1BB and CD3ろ intracellular domain, that is promising for further development and clinical investigation.

\section{Abbreviations}

CAR-T, chimeric antigen receptor $\mathrm{T} ; \mathrm{r} / \mathrm{r}$ B-NHL, relapsed or refractory $\mathrm{B}$ cell non-Hodgkin lymphoma; mPFS, median progression-free survival; scFv, single-chain variable fragment; $\mathrm{CRS}$, Cytokine release syndrome; ICANS, immune effector cell-associated neurotoxicity syndrome; ORR, overall response rate; $\mathrm{CR}$, complete remission; PR, partial response; SD, stable disease; DLT, dose-limiting toxicity; IFN, interferon; TNF, tumor necrosis factor; $C B A$, cytometric bead array; $M Z L$, marginal zone B-cell lymphoma; DLBCL, diffuse large $B$ cell lymphoma; FL, follicular lymphoma; ECOG, Eastern Cooperative Oncology Group; BTD, Baseline tumor burden; SPD, sum of the products of diameters.

\section{Declarations}

Ethics approval and consent to participate: The clinical study was approved by the Ethics Committee of Drug Clinical Trials of Peking University Cancer Hospital. This study was conducted in accordance with the Declaration of Helsinki, and all participants were informed of the possible risks and side effects of the therapy, and provided signed informed consent.

Competing Interests: The authors declare that they have no competing interests.

Potential conflicts of interest: HT, LX, JS, DY and HX are employed by Immunochina Pharmaceuticals Co., Ltd. HT and LX have equity ownership in Immunochina Pharmaceuticals Co., Ltd. The remaining authors declare no conflict of interest.

Funding: This study was supported by the National Nature Science Foundation of China (No. 81870154, 81972807, 81670187, 81970179 and 81700197); Beijing Natural Science Foundation (No. 7202025 and 7202026), Capital's Funds for Health Improvement and Research (No. 2018-1-2151 and 020-2Z-2157), Beijing Municipal Science \& Technology Commission (Z181100001918019), Beijing Municipal Administration of Hospitals' Ascent Plan (No. DFL20151001), and Science Foundation of Peking University Cancer Hospital (2020-5) $\llbracket$ Shandong Key Research and development Program ( No.2018GSF118097).

Author Contributions: Y.Z., H.T., L.X., S.Y., and J.Z. conceived and designed the study; Y.Z., W.X., Z.W., L.N., T.M., X.Y., P.L., Z.C., L.W., and D.L. performed clinical examinations; Y.Z., H.T., J.S., D.Y., H.X., B.B., L.X., and S.Y. analyzed and interpreted the data; L.X., and H.T. designed the CAR and prepared the CAR-T cell product; J.S., L.X., Y.Z., S.Y. and J.Z. wrote the manuscript; all authors read and approved the final manuscript. 
Acknowledgements: We are grateful for all patients who participated in our exploratory clinical studies.

Consent for publication: Not Applicable.

Availability of data and materials: The datasets used and/or analysed during the current study available from the corresponding author on reasonable request.

CONSORT guidelines: Our study adheres to CONSORT guidelines.

\section{References}

1. F. Bray, J.,et al., Global cancer statistics 2018: GLOBOCAN estimates of incidence and mortality worldwide for 36 cancers in 185 countries, CA Cancer J Clin, 68 (2018) 394-424.

2. W. Liu.,et al., Union for China Leukemia Investigators of the Chinese Society of Clinical, O. Union for China Lymphoma Investigators of the Chinese Society of Clinical, Burden of lymphoma in China, 2006-2016: an analysis of the Global Burden of Disease Study 2016, J Hematol Oncol, 12 (2019) 115.

3. D. Sun.,et al., Cancer burden and trends in China: A review and comparison with Japan and South Korea, Chin J Cancer Res, 32 (2020) 129-139.

4. N. Tomita., et al., Clinicopathological features of lymphoma/leukemia patients carrying both BCL2 and MYC translocations, Haematol-Hematol J, 94 (2009) 935-943.

5. C. Gisselbrecht.,et al., Salvage regimens with autologous transplantation for relapsed large B-cell lymphoma in the rituximab era, J Clin Oncol, 28 (2010) 4184-4190.

6. N. Mounier., et al., High-dose therapy and autologous stem cell transplantation in first relapse for diffuse large B cell lymphoma in the rituximab era: an analysis based on data from the European Blood and Marrow Transplantation Registry, Biol Blood Marrow Transplant, 18 (2012) 788-793.

7. A. Kallam., et al., Recent Advances in CAR-T Cell Therapy for Non-Hodgkin Lymphoma, Clin Lymphoma Myeloma Leuk, 19 (2019) 751-757.

8. J.S. Abramson., et al., Anti-CD19 CAR T Cells in CNS Diffuse Large-B-Cell Lymphoma, N Engl J Med, 377 (2017) 783-784.

9. Z.J. Roberts., et al., Axicabtagene ciloleucel, a first-in-class CAR T cell therapy for aggressive NHL, Leuk Lymphoma, 59 (2018) 1785-1796.

10. J.S. Qin., et al., Antitumor Potency of an Anti-CD19 Chimeric Antigen Receptor T-Cell Therapy, Lisocabtagene Maraleucel in Combination With Ibrutinib or Acalabrutinib, J Immunother, 43 (2020) 107-120.

11. J.S. Abramson., et al., Lisocabtagene maraleucel for patients with relapsed or refractory large B-cell lymphomas (TRANSCEND NHL 001): a multicentre seamless design study, Lancet, 396 (2020) 839852. 
12. F.L. Locke., et al., Phase 1 Results of ZUMA-1: A Multicenter Study of KTE-C19 Anti-CD19 CAR T Cell Therapy in Refractory Aggressive Lymphoma, Mol Ther, 25 (2017) 285-295.

13. S.J. Schuster., et al., Tisagenlecleucel in Adult Relapsed or Refractory Diffuse Large B-Cell Lymphoma, N Engl J Med, 380 (2019) 45-56.

14. D.W. Lee., et al., Current concepts in the diagnosis and management of cytokine release syndrome, Blood, 124 (2014) 188-195.

15. M.C. O'Leary., et al., FDA Approval Summary: Tisagenlecleucel for Treatment of Patients with Relapsed or Refractory B-cell Precursor Acute Lymphoblastic Leukemia, Clin Cancer Res, 25 (2019) $1142-1146$.

16. J.S. Abramson, Anti-CD19 CAR T-Cell Therapy for B-Cell Non-Hodgkin Lymphoma, Transfus Med Rev, 34 (2020) 29-33.

17. Z. Ying., et al., Parallel Comparison of 4-1BB or CD28 Co-stimulated CD19-Targeted CAR-T Cells for B Cell Non-Hodgkin's Lymphoma, Mol Ther Oncolytics, 15 (2019) 60-68.

18. X. Zhao., et al., Efficacy and Safety of CD28- or 4-1BB-Based CD19 CAR-T Cells in B Cell Acute Lymphoblastic Leukemia, Mol Ther Oncolytics, 18 (2020) 272-281.

19. K.A. Hay.,et al., Kinetics and biomarkers of severe cytokine release syndrome after CD19 chimeric antigen receptor-modified T-cell therapy, Blood, 130 (2017) 2295-2306.

20. A.C. Boroughs., et al., A Distinct Transcriptional Program in Human CAR T Cells Bearing the 4-1BB Signaling Domain Revealed by scRNA-Seq, Mol Ther, 28 (2020) 2577-2592.

21. O.U. Kawalekar.,et al., Distinct Signaling of Coreceptors Regulates Specific Metabolism Pathways and Impacts Memory Development in CAR T Cells, Immunity, 44 (2016) 712.

22. F.L. Locke., et al., Long-term safety and activity of axicabtagene ciloleucel in refractory large B-cell lymphoma (ZUMA-1): a single-arm, multicentre, phase 1-2 trial, Lancet Oncol, 20 (2019) 31-42.

23. T. Gargett., et al., Optimization of manufacturing conditions for chimeric antigen receptor $T$ cells to favor cells with a central memory phenotype, Cytotherapy, 21 (2019) 593-602.

24. D.L. Porter., et al., Chimeric antigen receptor T cells persist and induce sustained remissions in relapsed refractory chronic lymphocytic leukemia, Sci Transl Med, 7 (2015) 303ra139.

25. D. Sommermeyer., et al., Chimeric antigen receptor-modified T cells derived from defined CD $8+$ and CD4 + subsets confer superior antitumor reactivity in vivo, Leukemia, 30 (2016) 492-500.

26. D.C. Bishop.,et al., PiggyBac-Engineered T Cells Expressing CD19-Specific CARs that Lack IgG1 Fc Spacers Have Potent Activity against B-ALL Xenografts, Mol Ther, 26 (2018) 1883-1895.

27. C.J. Turtle., et al., CD19 CAR-T cells of defined CD4(+): CD8(+) composition in adult B cell ALL patients, Journal of Clinical Investigation, 126 (2016) 2123-2138.

28. C.J. Turtle., et al., Immunotherapy of non-Hodgkin's lymphoma with a defined ratio of CD8(+) and CD4(+) CD19-specific chimeric antigen receptor-modified T cells, Science Translational Medicine, 8 (2016). 
Figures

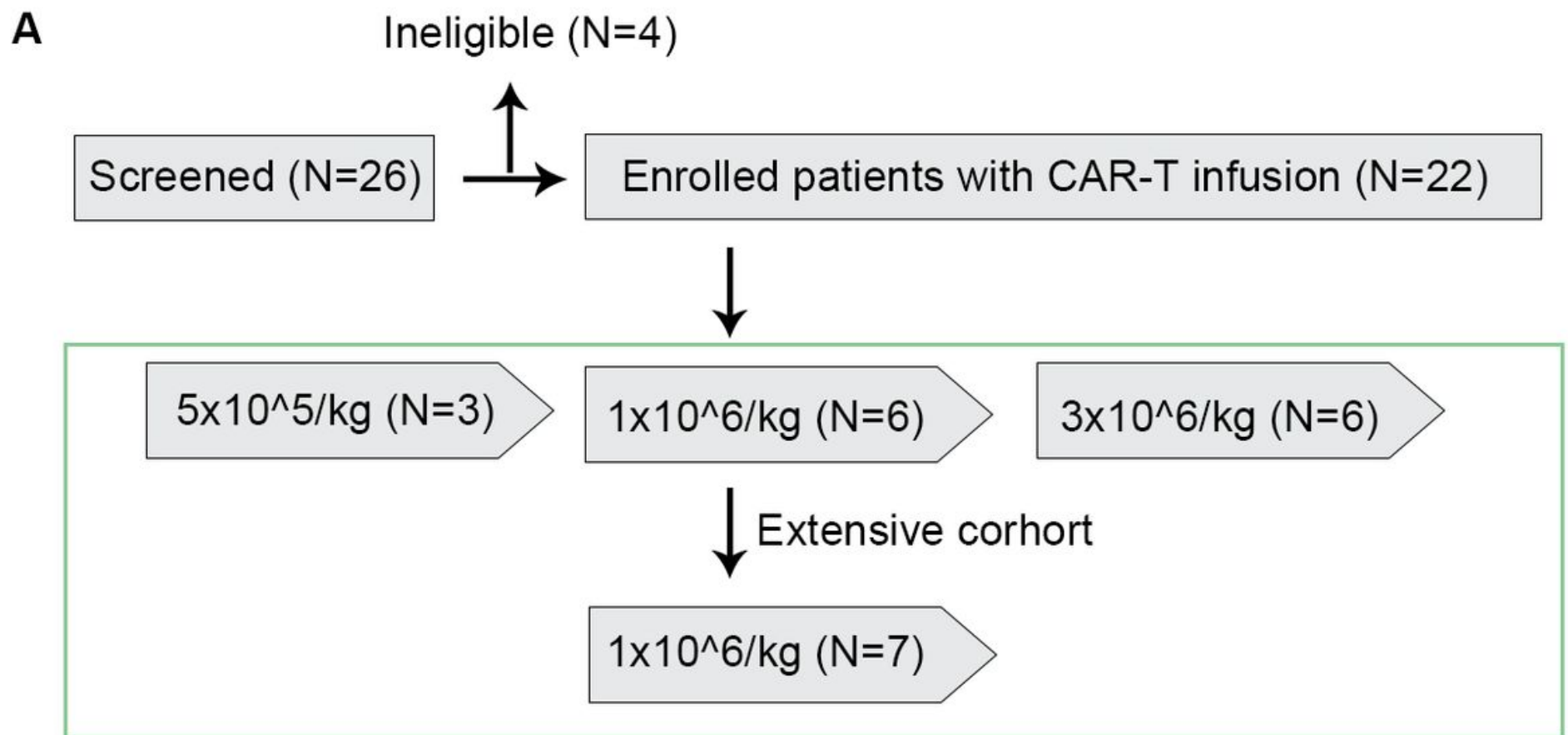

B

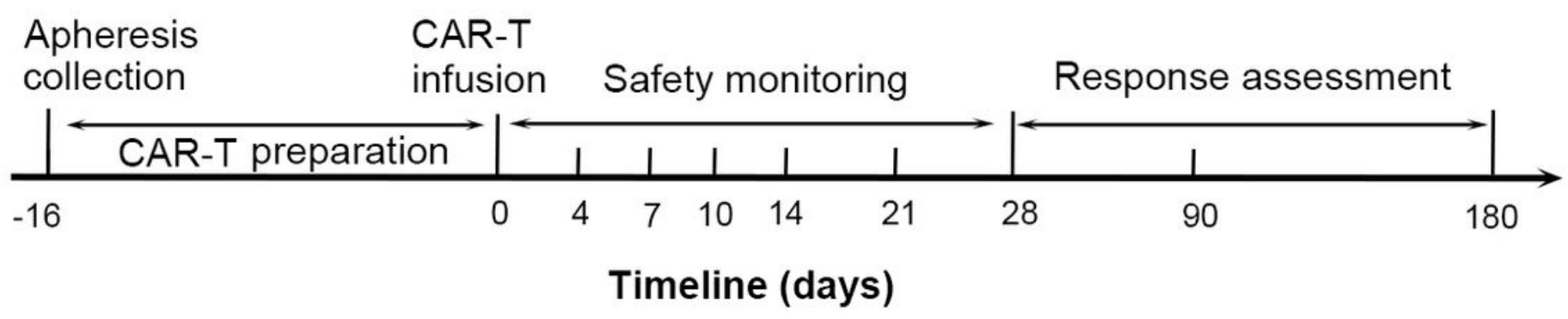

\section{Figure 1}

Schematic overview of the clinical trial. (A) Schematic overview of the patient cohorts; (B) Schematic overview of the clinical protocol. 
A

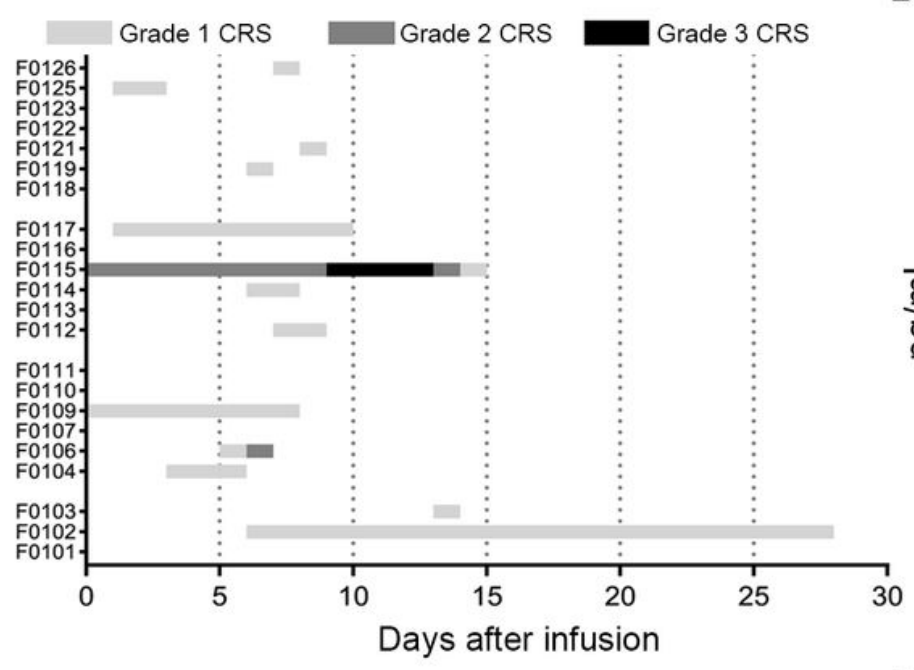

C

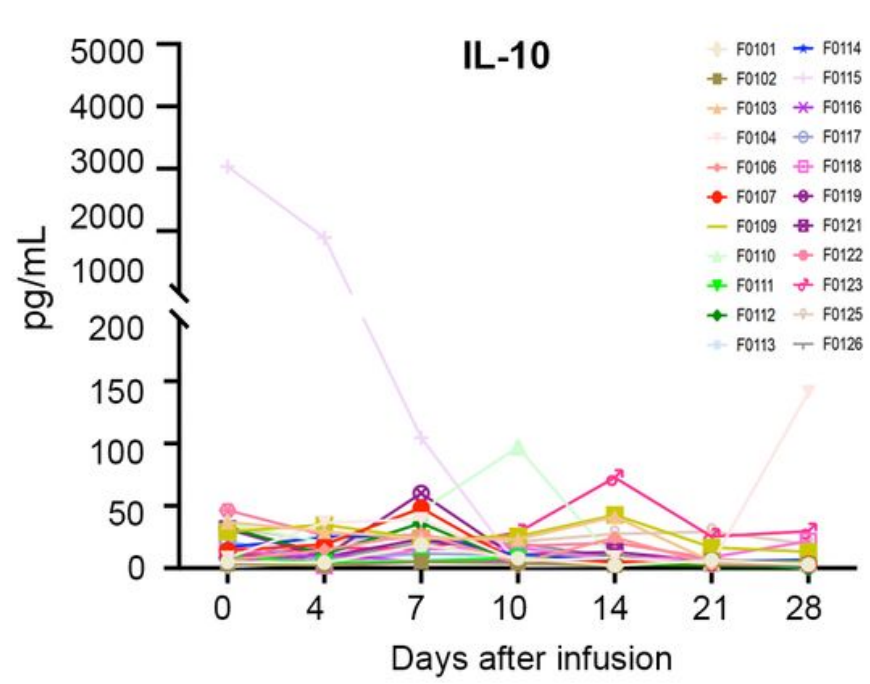

B

D

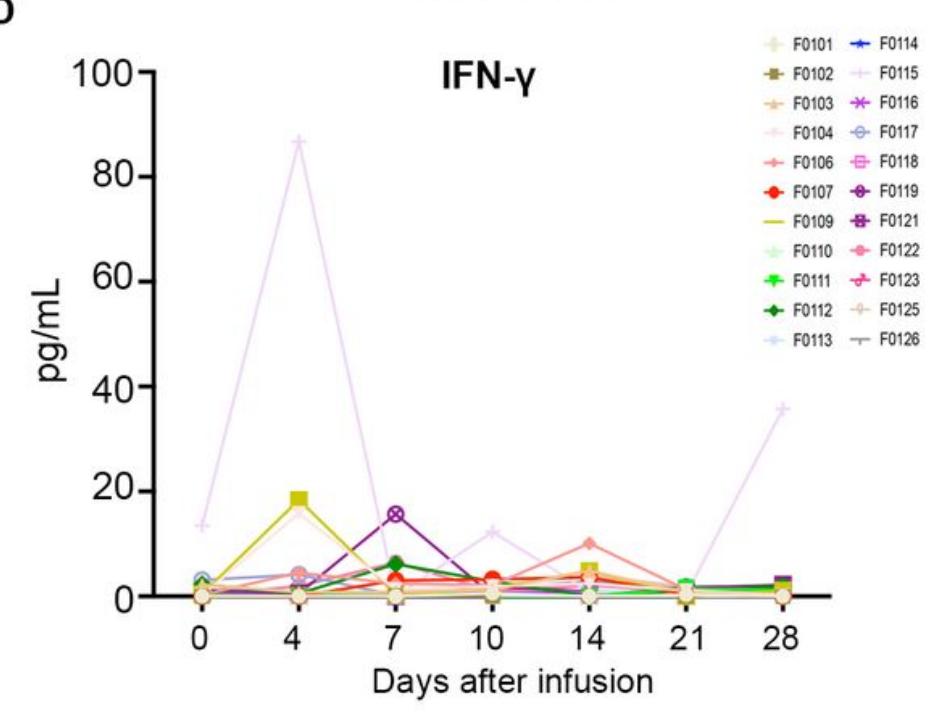

Figure 2

Evaluation of CRS and cytokine expression in patients after CAR-T cell infusion. (A) The CRS grade in each patient; (B-D) The time course of serum IL-6 (B), IL-10 (C) and IFN-Y (D) in 22 patients after CAR-T cell therapy. The first day of CD19 CAR-T cell infusion was regarded as day 0. 
A

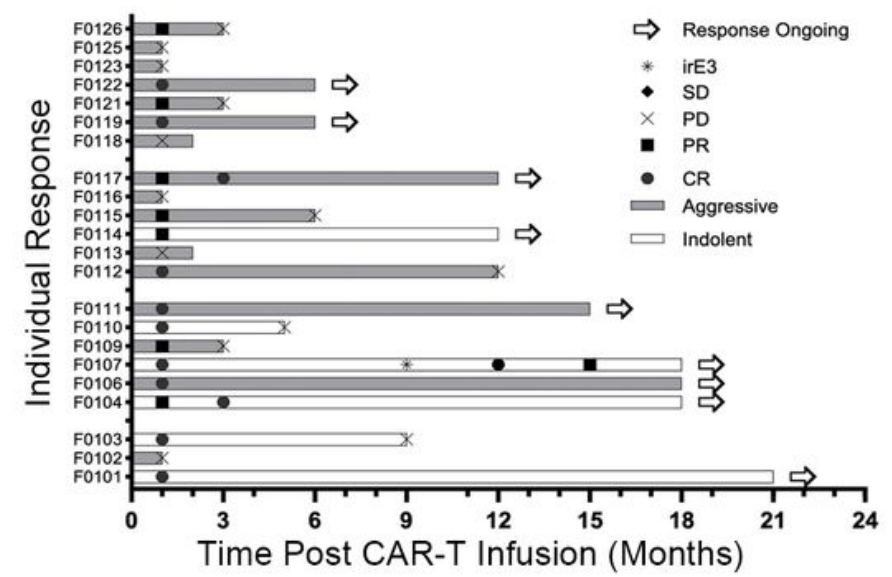

C

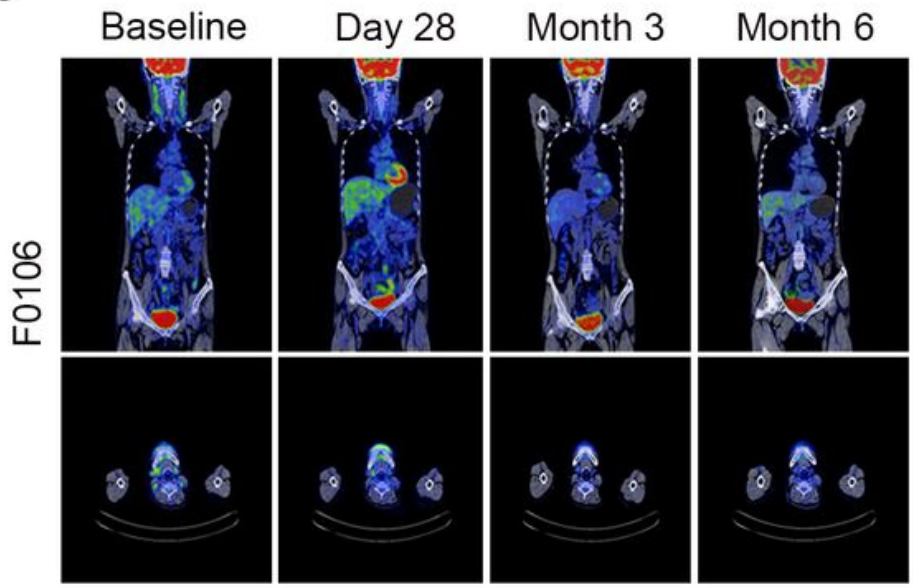

Crosssectional Neck

D

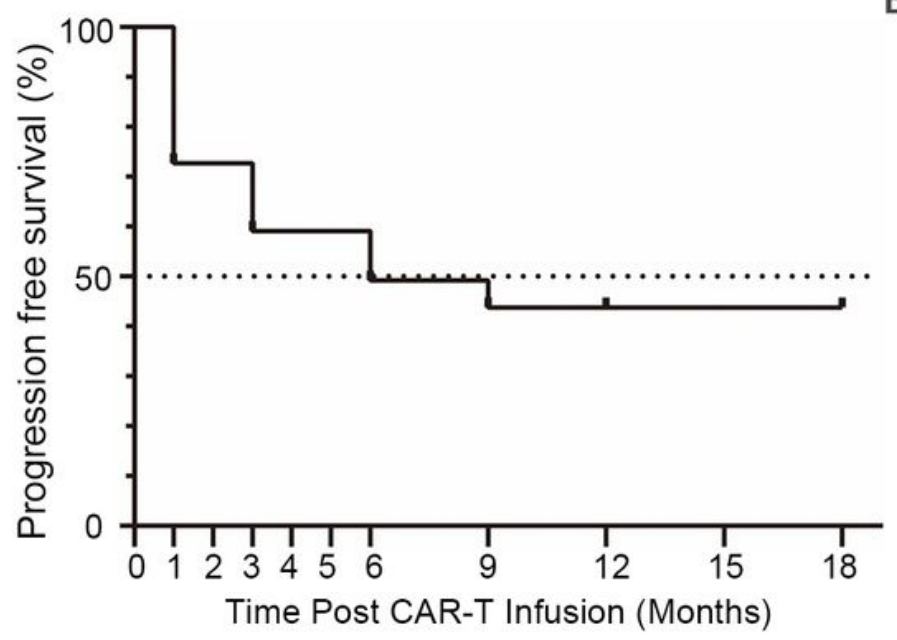

B

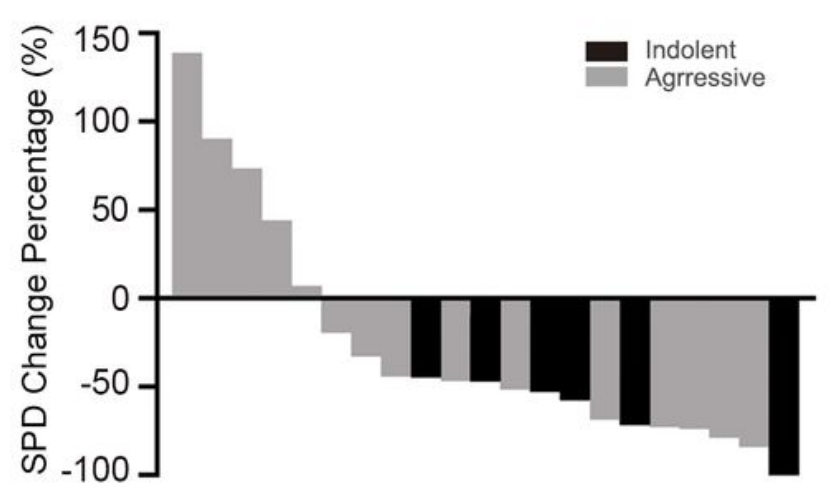

Individual Results at day 28
Baseline Day 28 Month 3 Month $6 \quad$ Month 12

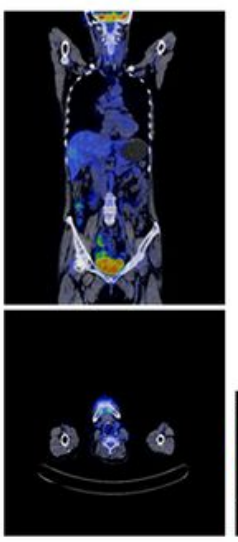

E

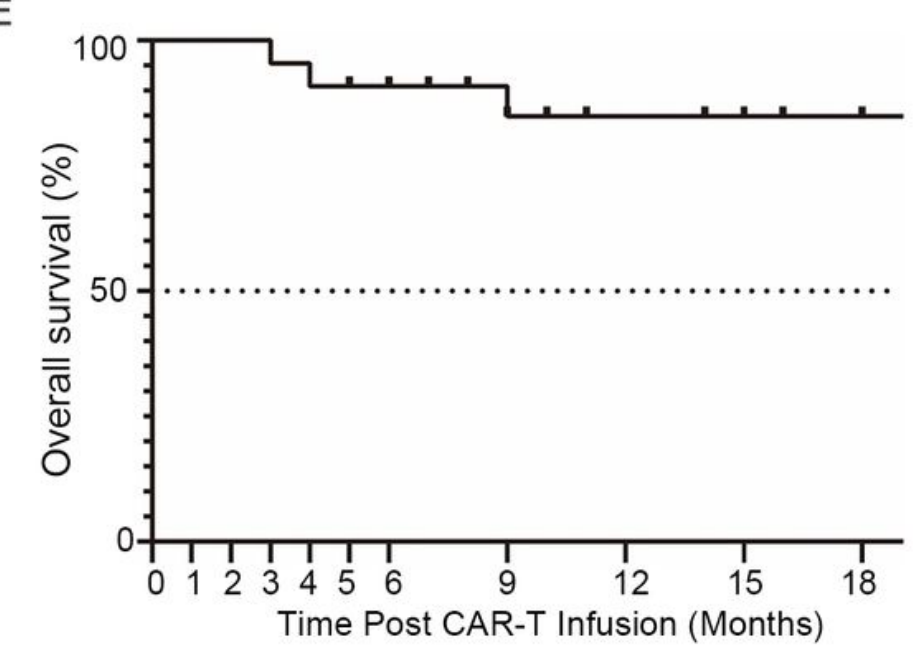

Figure 3

The clinical response of IM19 CAR-T cells in r/r NHL patients. (A) Clinical response in each patient after CAR-T cell infusion. IrE3: immune response 3; SD: severe disease; PD: progressive disease; PR: partial remission; CR: complete response; SPD: sum of the products of diameters. (B) Evaluation of the tumor burden of 21 patients after CAR-T cell infusion. (C) PET-CT images of two presentative r/r NHL patients 
who showed an ongoing CR after IM19 CAR-T therapy. (D) Progression-free survival rate of patients after IM19 infusion. (E) Overall survival rate of patients after IM19 infusion.

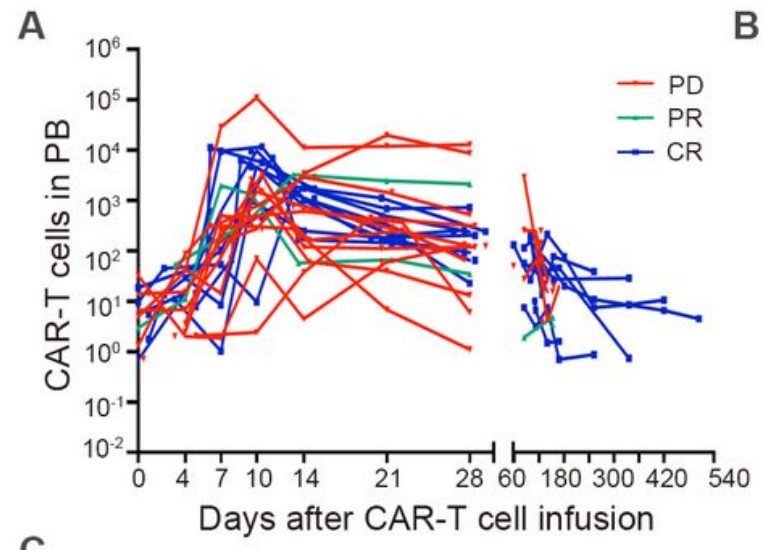

B

C
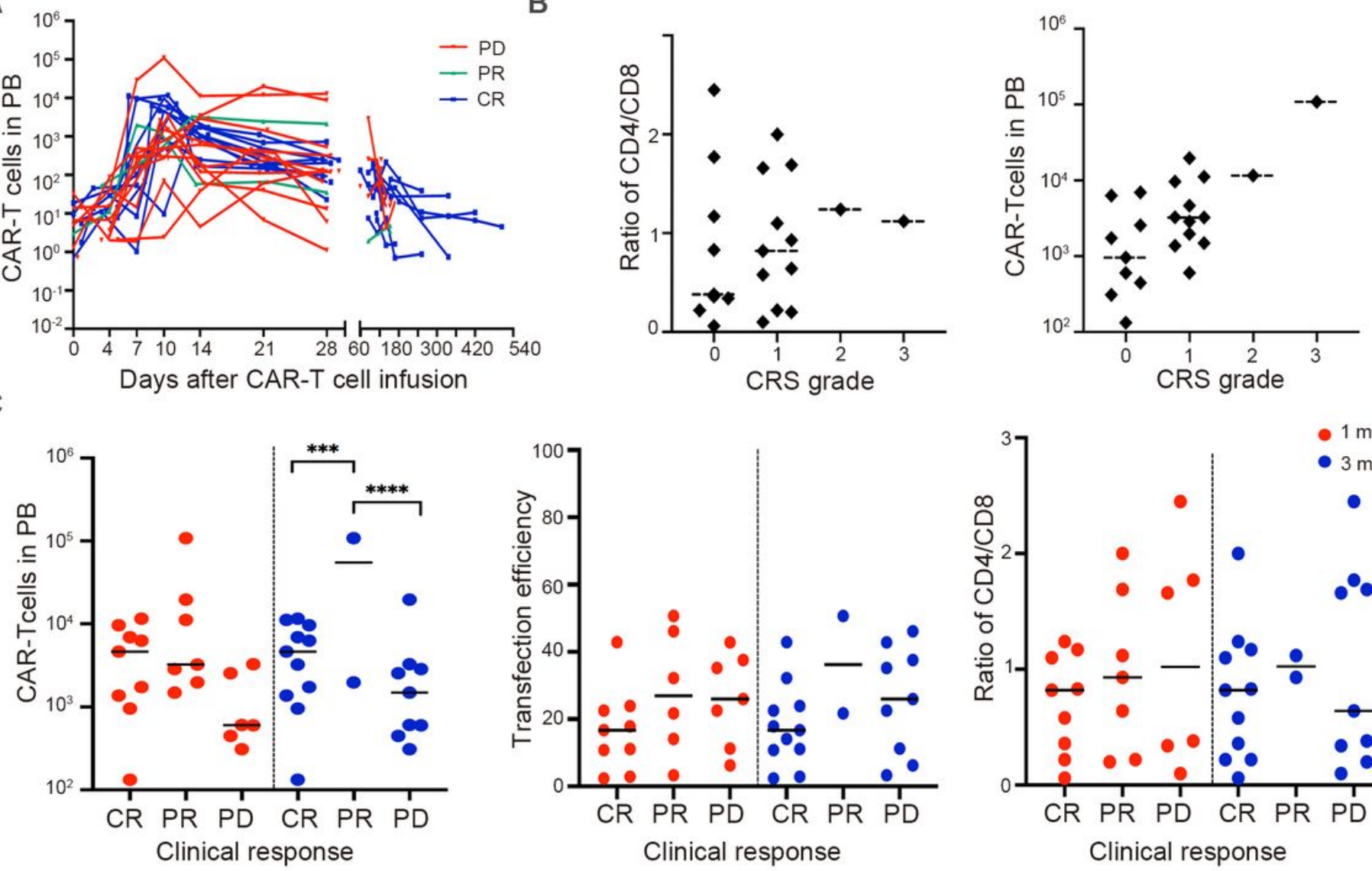

D

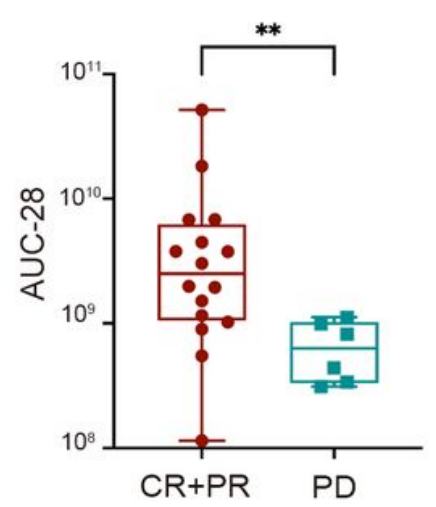

Clinical response
E

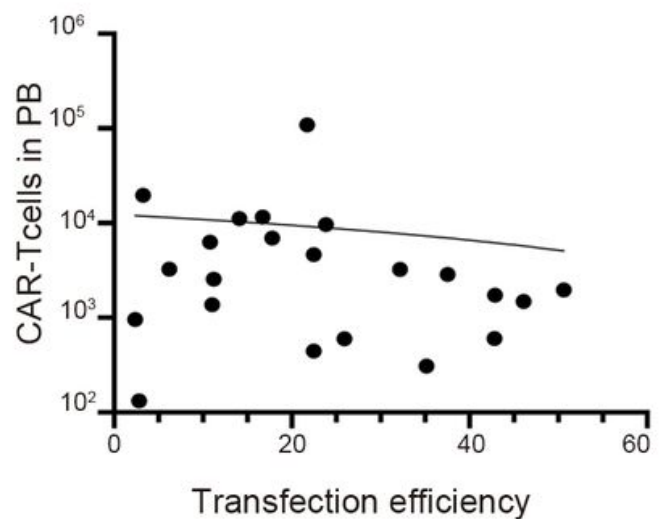

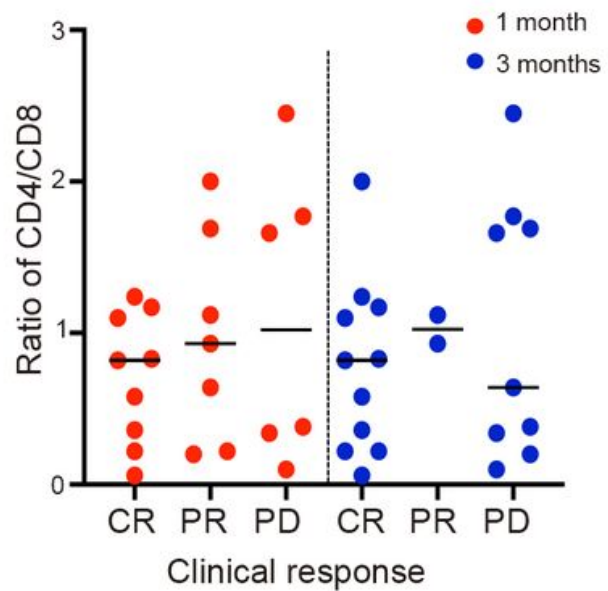

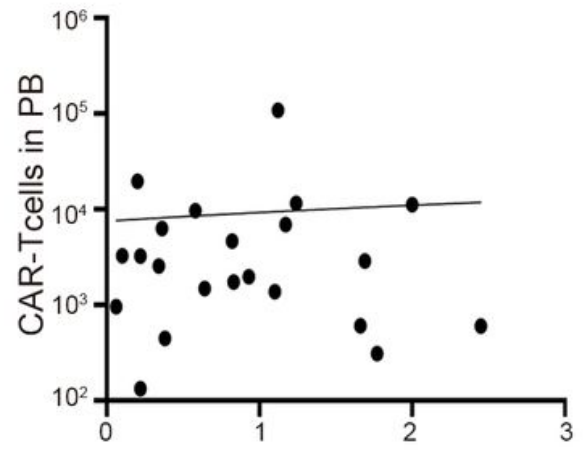

Ratio of CD4/CD8

\section{Figure 4}

Kinetics of IM19 CAR-T cells in patients and correlation with clinical outcomes. (A) In vivo kinetics of IM19 CAR-T cells in different response groups. (B) Correlation analysis of CD4/CD8 ratio and CAR-T cell peak in peripheral blood and the CRS grade. (C) Correlation analysis of CAR-T cell peak in peripheral blood (left), transduction efficiency (median), and the ratio of CD4:CD8 (right) with different clinical response groups at 1 month and 3 months. (D) Comparison of the AUC-28 size between CR+PR groups and PD groups. AUC-28: area under the curve of CAR-T cell expansion from day 0 to 28. (E) Correlation of CAR-T cells in peripheral blood with transduction efficiency (left) or with ratio of CD4:CD8 (right). 


\section{Supplementary Files}

This is a list of supplementary files associated with this preprint. Click to download.

- supplementaryfile.docx 